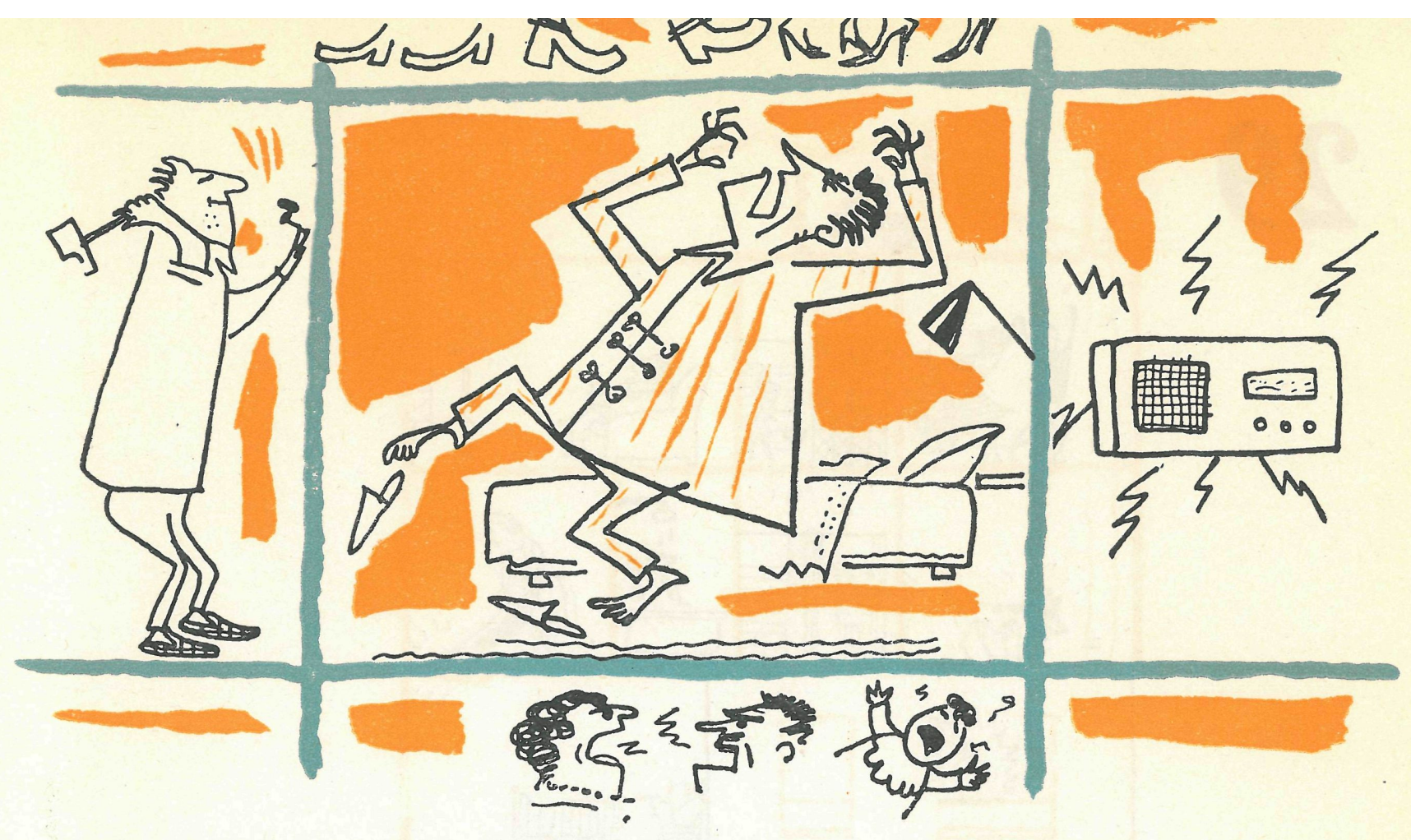

$340-3$

\title{
los ruidos en las edificaciones IV
}

J. M. TOBIO, Dr. en Química Industrial

\section{vibraciones y ruidos industriales}

\section{5.- Como se transmite el ruido}

En el problema acústico de una edificación hay dos aspectos bien diferentes. Primero, el aislamiento y corrección de un edificio ya construído en el que hay que respetar una serie de servidumbres (estructura, muros, forjados, canalizaciones, ventanas, puertas, etc.).

Este es un caso en el que debe contarse de antemano con unos resultados acústicos mediocres - limitados, e incluso llegar a la imposibilidad práctica de resolver los problemas planteados. Las razones son obvias.

En segundo lugar existe el caso de un edificio de nueva planta, que tiene, en general, solución. No hay más que adoptar las medidas adecuadas, tal como se explica en los capítulos correspondientes de esta obra. Si los niveles sonoros externos no son extremadamente elevados $(>110$ fonios), o si los niveles admisibles en los distintos locales no son demasiado bajos ( $<25$ fonios) (ver 14), la solución no entraña frecuentemente más que un gasto extra perfectamente soportable.

Los sonidos pueden llegar al interior de los locales siguiendo diversas trayectorias, la mayoría de las cuales están señaladas en el esquema de la figura 29. Pueden entrar a través de aberturas (ventanas y puertas abiertas, grietas alrededor de los tubos, conducciones eléctricas, agujeros de ventilación, etc.).

También pueden entrar por vibración diafragmática de tabiques, muros y suelos. Finalmente, pueden llegar por impacto directo (caída de objetos sobre el suelo, ruido de sillas, pasos, etc.) (ver figura 29).

Respecto al aislamiento de los ruidos transmitidos por el aire (aberturas) y a través de tabiques y suelos, los capítulos II y III muestran la forma de atacar estos problemas. En los "Apéndices", al final del capítulo $\mathrm{V}$ se incluyen algunos datos numéricos sobre diversos sistemas constructivos usuales. 


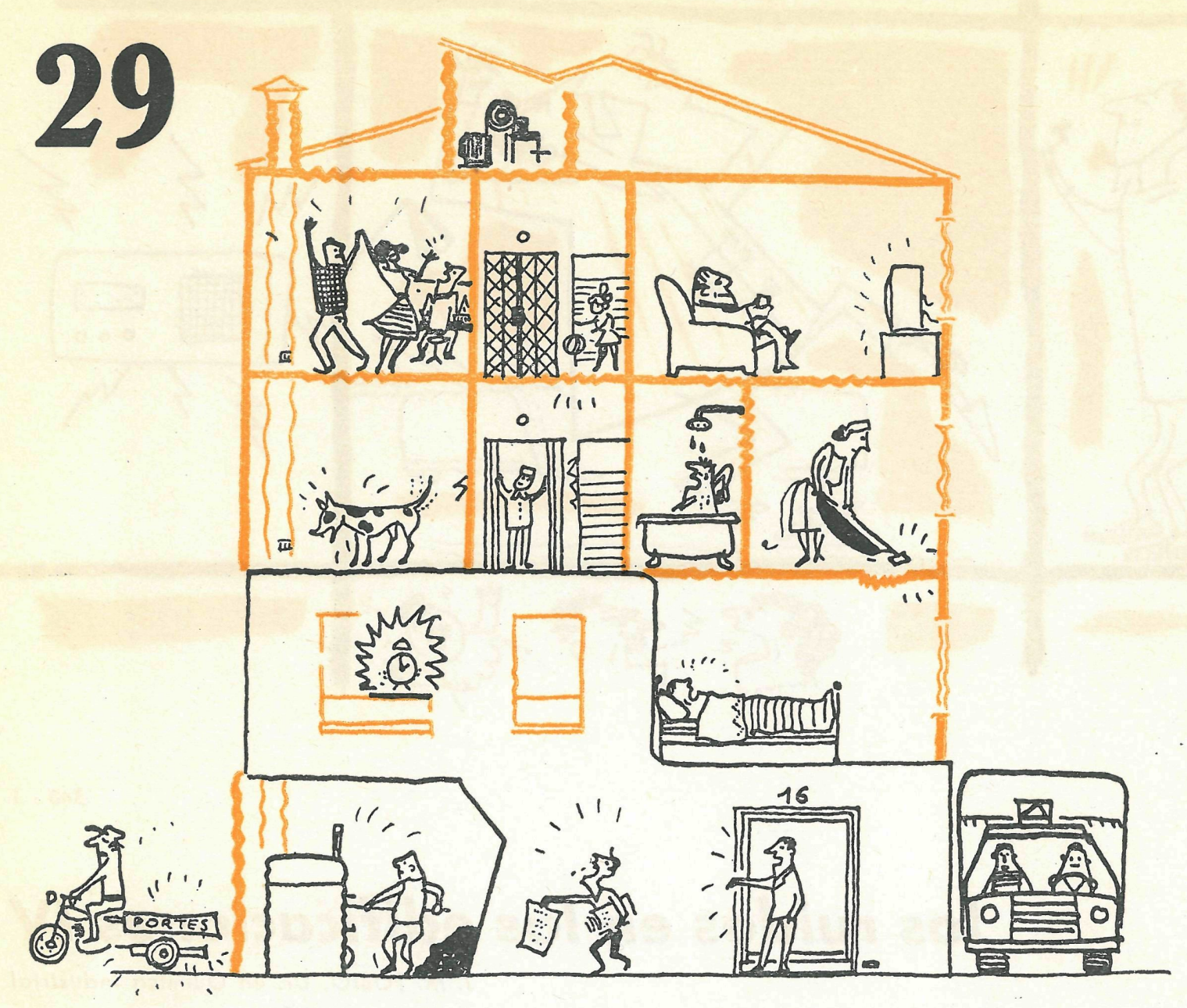

\section{6.-Ruidos producidos por aparatos}

Aunque en 13 se hizo mención a los ruidos transmitidos por los sólidos, el problema de las vibraciones y ruidos industriales exige un tratamiento separado.

Tenemos, en primer lugar, los choques o trepidaciones. Son perturbaciones mecánicas directas, de duración generalmente breve, que provocan una vibración de los materiales, de espectro muy complejo y que, en la mayoría de los casos, pueden convertirse en ondas sonoras perjudiciales. La construcción discontinua y el revestimiento de pavimentos con materiales "blandos" son los remedios generales para este tipo de ruidos.

Un pavimento de caucho, fieltro o moqueta puede provocar una atenuación de 7 a 15 db para los choques de pasos o movimientos de mobiliario.

El peligro de las perturbaciones por choques directos disminuye al aumentar el peso de las estructuras.

Otro tipo de ondas de choque es el provocado por las tuberias, generalmente las de conducción de agua. Si el régimen de paso del líquido por los tubos no es laminar sino turbulento, pueden originarse ondas de choque del flúido contra las paredes del tubo. Esto suele ocurrir cuando la velocidad de paso del agua por las tuberías es superior a $2,5 \mathrm{~m} / \mathrm{s}$.

El cierre más o menos brusco de los grifos provoca una discontinuidad de presión que se traduce por una. "onda de choque", conocida generalmente como "golpe de ariete". Este choque perturba las tuberías y da lugar a un ruido. Se puede combatir eficazmente mediante un dispositivo a base de manguitos elásticos dispuestos en la tubería.

Existen en el comercio algunos tipos especiales de grifos "anti-turbulencia", llamados también grifos silenciosos, que deben utilizarse cuando existe el peligro de estas perturbaciones (fig. 30 ).

Otros tipos de ruidos domésticos, tales como los producidos por aspiradores eléctricos, ventiladores, etc. no requieren consideración especial. Pueden combatirse con los medios usuales, tal como se expresó en los capítulos II y III. 


\section{7.-Vibraciones}

Las diferentes máquinas que pueden existir en un edificio-motobombas para elevación de aguas, motores de ascensores y montacargas y otrospueden producir, aparte del ruido normal originado por el movimiento de las partes móviles, vibraciones sobre los materiales en que se apoyan.

Si bien las intensidades de los sonidos de impacto (choque) se miden como los demás sonidos, en fonios, para las vibraciones se ha adoptado una unidad especial, que es el PAL, definida por:

$$
\text { Int. de la vibración }=10 \log \left(\frac{u}{u_{0}}\right)^{2} \text { pal, }
$$

en la que $u$ es el valor eficaz de la velocidad de la onda vibratoria y $u_{0}$ la velocidad más baja perceptible, que se ha establecido como $0,0312 \mathrm{~cm} / \mathrm{s} *$.

Para tener una orientación sobre los valores usuales de pal dados por [13], y que pueden medirse con un "medidor de pal" (fig. 31), indicamos en la tabla siguiente los que pueden hallarse en la práctica:

\begin{tabular}{|c|c|}
\hline Talor umbral & 0 pal \\
\hline 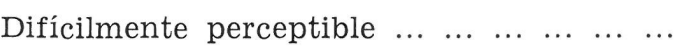 & 5 pal \\
\hline $\begin{array}{llllllll}\text { eptible } & \ldots & \ldots & \ldots & \ldots & \ldots & \ldots\end{array}$ & \\
\hline & \\
\hline able & \\
\hline
\end{tabular}

En los edificios, las vibraciones continuas procedentes de máquinas no deben exceder de 5 pal. En el caso de fenómenos transitorios, por ejemplo, el paso de un pesado camión, pueden aceptarse hasta 20 pal.

La forma de rebajar las vibraciones fuertes a valores admisibles se basa siempre en usar soportes elásticos cuyas características sean tales que respondan mal a las solicitaciones periódicas que sufren. Para que ello ocurra deben cumplirse estrictamente las dos condiciones siguientes:

a) Que el sistema de apoyo o suspensión sea verdaderamente vibrante, es decir, que no se cargue más allá del límite elástico. Muy a menudo se descuida esta característica cuando se montan máquinas sobre goma, corcho, lana de vidrio, resortes, etc., y se coloca una superficie de apoyo insuficiente con respecto al peso de la máquina.

b) Que la frecuencia propia del sistema sea efectivamente mucho más baja que la frecuencia de la perturbación que se quiere combatir. Si ello no ocurre así, los resultados son desastrosos, mucho peores que no utilizando apoyo o suspensión alguno. Por dicha razón es preciso conocer de antemano tanto la frecuencia del aparato que vibra como la propia de los soportes.

Respecto a disposiciones prácticas, las mismas casas que venden los materiales elásticos dan normas para su montaje. La figura 32 muestra un ejemplo de montaje anti-vibrante de una máquina sobre una masa que, a su vez, está suspendida elásticamente del suelo. La presencia de esta masa es necesaria para rebajar la frecuencia de resonancia del sistema de suspensión, tal como se dijo en $b$ ).

El caso más sencillo es el montaje de una máquina de masa M (en la que se incluye la plataforma o masa auxiliar suspendida, como en la figu-

* Entendiendo por velocidad la derivada del desplazamiento del objeto que vibra, con relación al tiempo:

$$
u=\frac{d x}{d t} \mathrm{~cm} / \mathrm{s}
$$
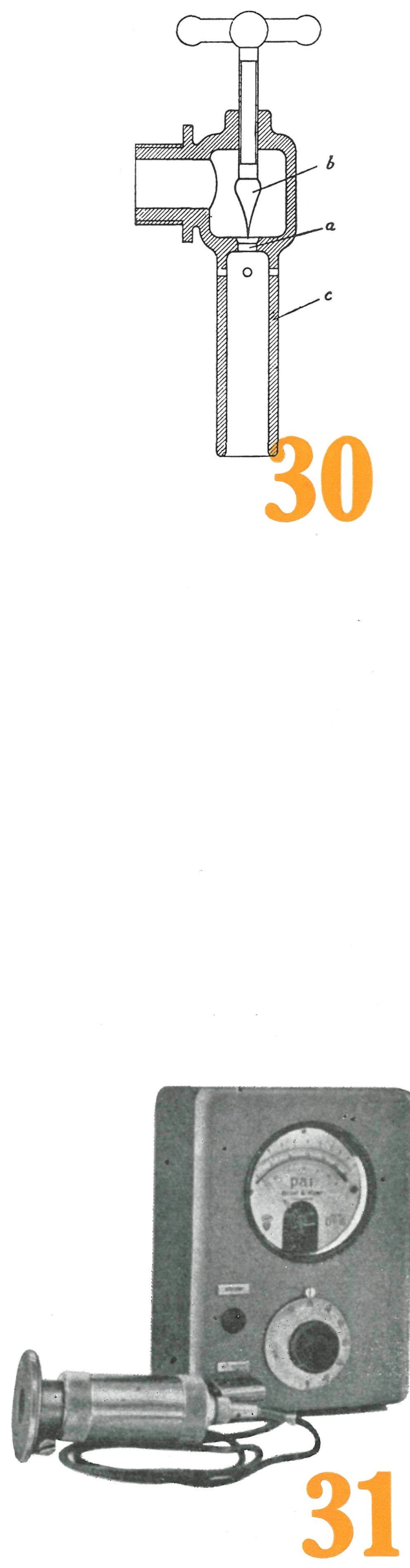

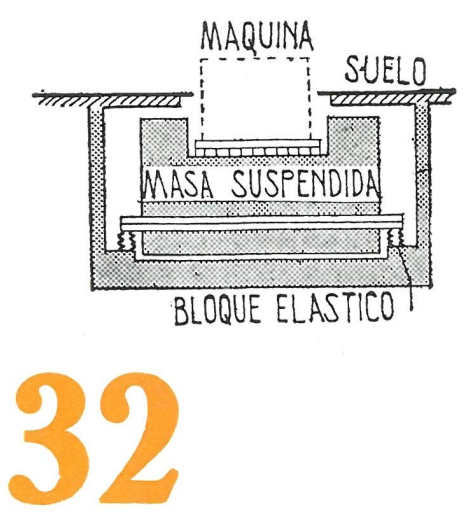

ra 32) sobre una lámina elástica de caucho o corcho. La frecuencia de resonancia del sistema de masa $\mathrm{M}$ viene dada por la fórmula:

$$
f=\frac{1}{2 \pi \sqrt{\frac{\mathrm{M}}{\mathrm{K}}}} \text { (ciclos/minuto) }
$$

en la que $\mathrm{K}$ es la constante de rigidez del sistema, es decir, la fuerza requerida para producir un desplazamiento unidad en el sistema de montaje.

Conociendo $f$ y la frecuencia de vibración de la máquina es fácil calcular el porcentaje de reducción de la vibración por medio de las gráficas de la figura 33. Cuando no se conoce la frecuencia propia de resonancia, puede utilizarse la "deformación estática" del sistema elástico. Esta es, en mm, la deflexión ocasionada en el soporte elástico cuando se coloca encima la masa M (máquina + base).

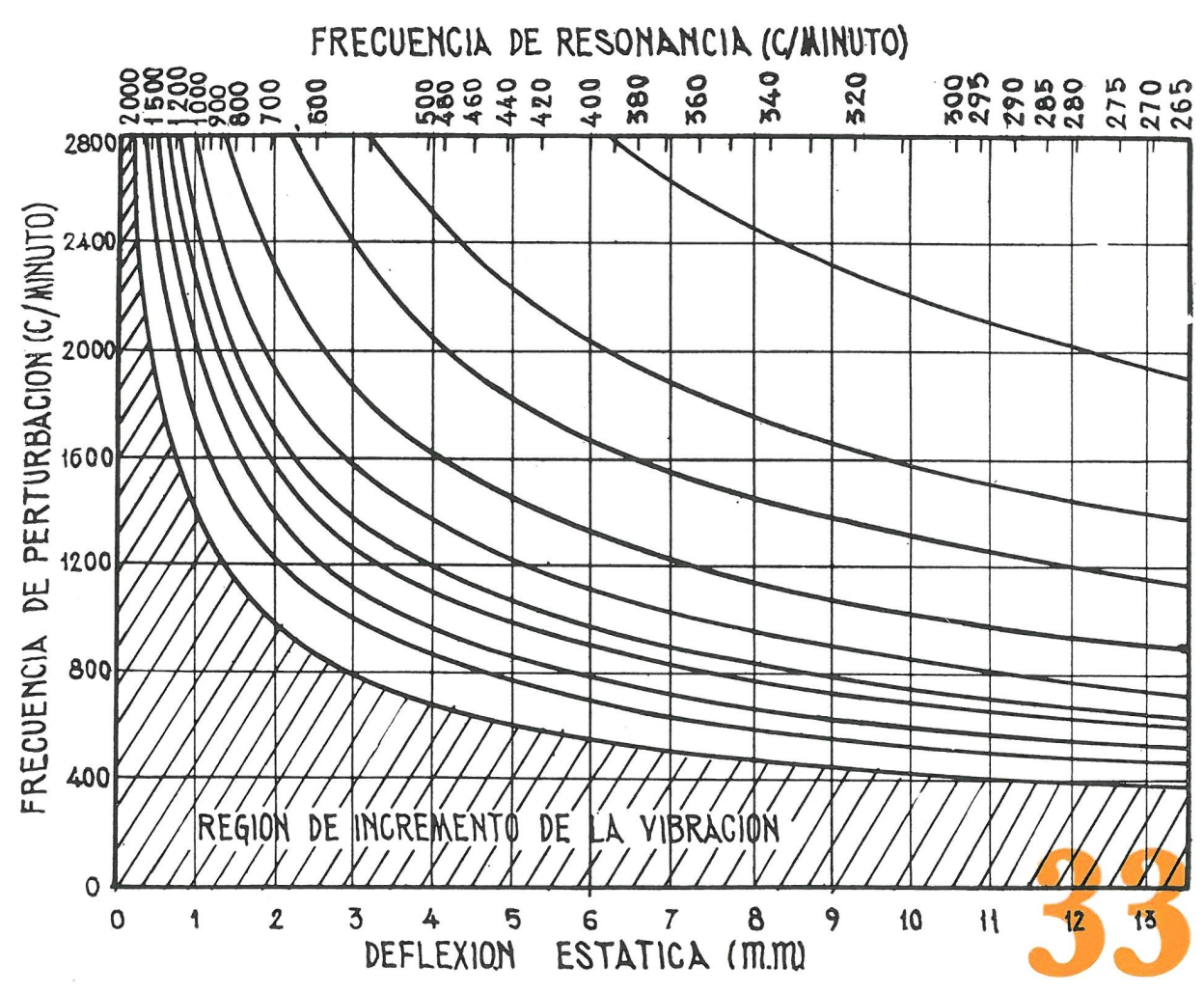

Ejemplo: Un motor que gira a 1.200 r.p.m. (fig. 34) se coloca sobre unos tacos de goma de sección suficiente para no sobrepasar el límite elástico del caucho [observación a]. Al colocar el motor, el caucho se deforma $3,8 \mathrm{~mm}$ (disminución aparente de grosor). Las curvas de la figura 33 nos muestran que, con estos valores, la disminución de vibración provocada por la suspensión es del orden de $80 \%$.

Véanse en las gráficas las dos zonas de reducción y aumento de la vibración a que se aludía en $b$ ). Si no se conoce la deformación estática del soporte y sí la frecuencia propia de resonancia del sistema, utilícese la escala superior de la figura 33. 


\section{8.-Ruidos en los sistemas de ventilación y acondicionamiento}

Un sistema de ventilación o acondicionamiento bien proyectado debe funcionar lo suficientemente silencioso como para no molestar en absoluto. Las tuberías de conducción de aire del sistema no deben servir, por otra parte, para transmitir las conversaciones y ruidos de un local a otro. Una regla práctica es que el nivel de ruidos resultante del sistema de ventilación deberá estar 5 db por debajo del nivel de ruidos aceptable para la habitación considerada (ver 14).

Los principales puntos a tener en cuenta para conseguir que el ruido creado por un sistema de acondicionamiento sea despreciable comparado con los demás ruidos de una vivienda son los que a continuación se indican:

1) Una elección e instalación adecuadas de motores, ventiladores y rejillas.

2) Un diseño aerodinámico de los sistemas de circulación de aire para evitar la turbulencia.

3) Aplicación de absorbentes en los conductos y equipos.

Cuando las exigencias acústicas son muy altas (como puede ocurrir en hospitales y estudios de radio), los sistemas de ventilación deben ser muy elaborados y se necesitarán filtros acústicos especiales.

Las principales fuentes de ruido en los sistemas de ventilación son los motores y ventiladores (soplantes), la turbulencia causada por el flujo del aire y los sonidos de origen externo que entran en el sistema a través de las paredes de los conductos o por la boca de salida de los mismos hacia las habitaciones.

Los motores y ventiladores pueden ocasionar ruidos transmitidos a través del aire o vibraciones por sólidos (ver 17).

Si $R$ es el número de revoluciones por minuto de un ventilador, aparece un sonido predominante ("frecuencia de las aspas") debido al rotor, cuya frecuencia es:

$$
\text { "Frecuencia de las aspas" }=\frac{N \cdot R}{50} \text { (ciclos/segundo) }
$$

en donde $\mathrm{N}$ es el número de aspas del ventilador. Para un ventilador a 3.500 r.p.m., con 6 aspas, la frecuencia predominante será de $420 \mathrm{c} / \mathrm{s}$.

Cuando se disponga un sistema de atenuación o filtro en los conductos de aire, habrá que tener muy en cuenta la frecuencia dada por [15].

En términos generales, el tratamiento de los conductos de aire ha de ser tal que se provoque el mayor amortiguamiento posible a lo largo de] recorrido de los mismos, sin interponer obstáculos a la circulación del aire. Este tratamiento, por otra parte, servirá también para debilitar los sonidos que puedan penetrar del exterior en el interior de los conductos.

Cuando se recubren las caras interiores de un conducto con un material absorbente (fig. 35) se logra una cierta atenuación, la cual viene dada en $\mathrm{db} / \mathrm{m}$ de longitud del conducto. Esta atenuación es dependiente de la frecuencia porque lo es $\alpha$ (ver 6), coeficiente de absorción del recubrimiento.
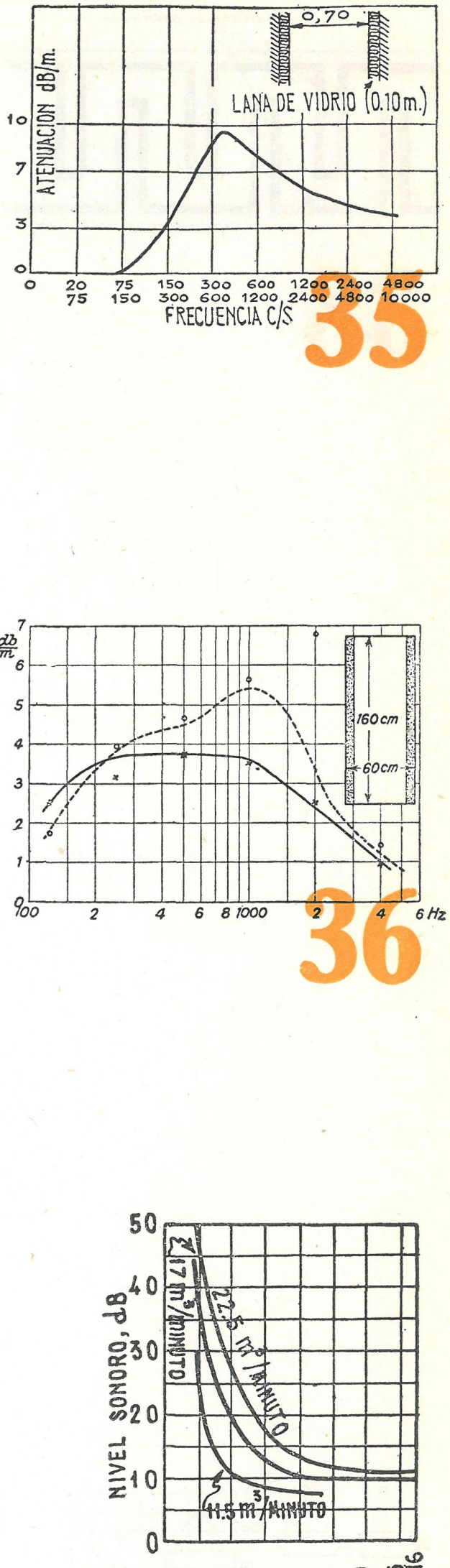

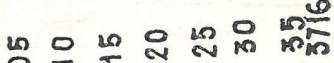
$\dot{0} \dot{0} \dot{0} \dot{0} \dot{0} \dot{0}$ AREA DE LA REJULLA $\mathrm{m}^{2}$ 

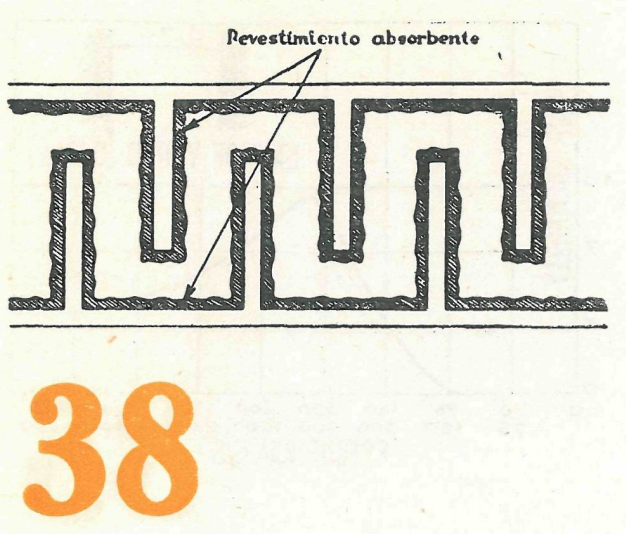

De un modo general puede calcularse la atenuación total de un conducto de $\mathrm{L}$ metros de largo y sección $\mathrm{S}$ en $\mathrm{cm}^{2}$, por la fórmula:

$$
N=21 \cdot \alpha \cdot \frac{P \cdot L}{S}(d b)
$$

en la que $\mathrm{P}$ es el perímetro, en $\mathrm{cm}$, de la sección recubierta. El coeficiente $\alpha$ es el mismo utilizado en 6.

Resulta claro que en la elección del material de recubrimiento habrá que tener presente que su coeficiente $\alpha$ sea lo más próximo posible al valor de la "frecuencia predominante" dado por [15]. De este modo se logrará la máxima absorción o atenuación.

Por otra parte, este material absorbente de recubrimiento-dado el lugar en que habrá de ser colocado-deberá ser incombustible, resistente a la humedad (incluso en caliente) y ofrecer poca resistencia al paso del aire.

En el comercio se encuentran "mantas" de lana de vidrio, lana de roca, conjuntos metal perforado-absorbente, etc., que cumplen estas finalidades.

El efecto de la frecuencia sobre la atenuación en $\mathrm{db} / \mathrm{m}$ de un conducto recubierto puede apreciarse en la fig. 36, en la que la línea llena corresponde a un recubrimiento de lana de roca de $8 \mathrm{~cm}$ de espesor y la curva de puntos a un absorbente con membrana tipo Bekesy (ver 7).

A pesar de estas disposiciones es muy difícil pasar de 10 s $6-7 \mathrm{db} / \mathrm{m}$ en el valor de la atenuación. Cuando se requieren valores más elevados hay que apelar al empleo de filtros (ver 19).

Existen otras muchas variantes en el tratamiento de los conductos, tales como el empleo de pantallas, la división del conducto en varios de menor sección, utilización de "rellenos" en forma helicoidal, y otros cuyo estudio nos separaría bastante de los límites impuestos a este trabajo.

Otra fuente de entrada de ruidos en las habitaciones la constituyen las rejillas terminales de los conductos en las paredes o techo. Para su proyecto deberá tenerse en cuenta que el ruido producido por la salida del aire (caliente o frí) aumenta cuando crece la velocidad de flujo. Las gráficas de la figura 37 relacionan el ruido producido con el área y la velocidad de salida del aire.

Las gráficas se aplican para cualquier tipo de orificio de salida: nido de abeja, rejilla con pantallas, deflectores, etc., siempre que se tengan en cuenta los valores reales de la sección y de la velocidad de flujo del aire.

\section{9.-Filtros acústicos}

Cuando las atenuaciones encontradas en un conducto de ventilación son inferiores a los valores requeridos para que el sistema no produzca ruidos inaceptables, es menester cortar los conductos en determinados puntos e intercalar filtros acústicos.

Estos filtros se ejecutan según tres directrices diferentes: disposiciones geométricas estudiadas de tal forma que, para un recorrido longitudinal dado "aparente", supongan un recorrido interior mucho más grande y una superficie absorbente también mayor. Así son los flltros en zigzag, helicoi- 
dales, etc., bastante eficaces a frecuencias medias y elevadas. Se pueden lograr atenuaciones de hasta $20-25 \mathrm{db} / \mathrm{m}$. La figura 38 es un ejemplo sencillo.

Otro sistema práctico es crear desadaptaciones de impedancia acústica (ver 6) mediante cambios bruscos de sección (un sistema parecido al silenciador de los automóviles). La figura 39 muestra un filtro doble semiselectivo de este tipo con las curvas de atenuación correspondientes. Obsérvese el especial comportamiento para determinadas frecuencias. Pueden alcanzarse atenuaciones de 30-40 db.

Por último, existen los filtros a resonancia, también selectivos. En éstos la sección del conducto no cambia, pero existen zonas perforadas que se comunican con espacios cerrados mayores o con materiales absorbentes.

Del dimensionado de estos dispositivos, diámetro de los orificios, etc., dependerá la frecuencia de resonancia (máxima atenuación) que, como siempre, deberá hacerse coincidir con la dada por la fórmula [15].

La figura 40 muestra la atenuación de una célula elemental de este último tipo. En la figura 41 puede verse el aspecto práctico de un filtro acústico a resonancia para un tubo de $100 \mathrm{~mm}$ de diámetro.
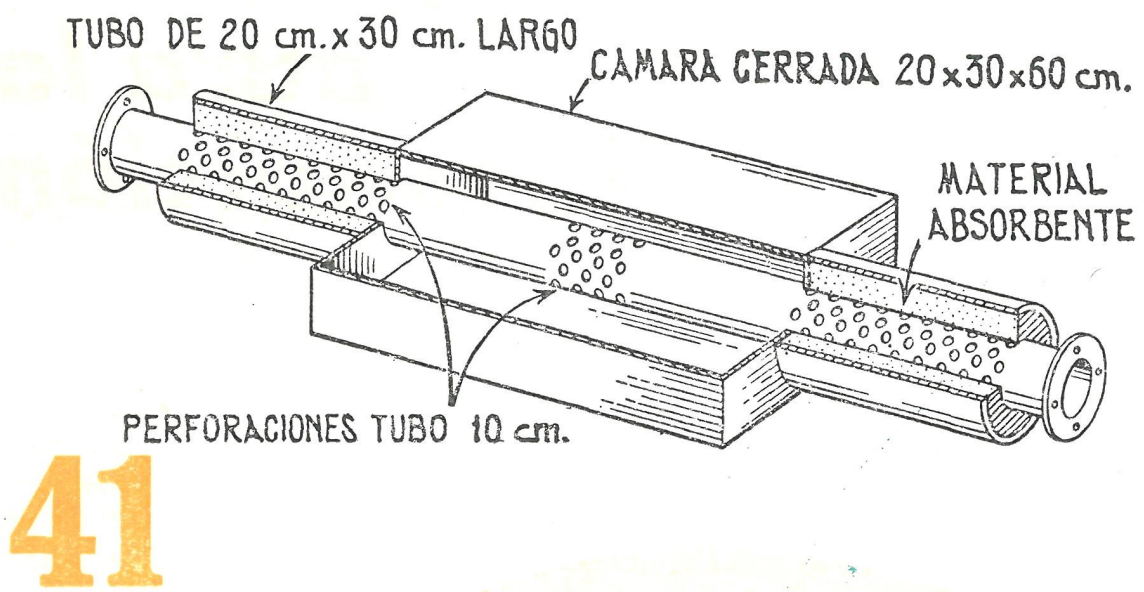

En la práctica es sumamente difícil el cálculo teórico de esta clase de filtros. Generalmente se construyen "a la medida" y luego se experimenta sobre ellos. Por otra parte, como veremos en el capítulo siguiente, es sencillo hacer medidas y comprobaciones experimentales con toda clase de dispositivos acústicos.
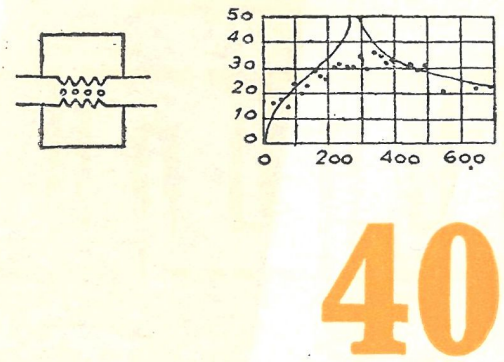